\title{
Clinical and radiographic outcomes of oblique lateral lumbar interbody fusion and minimally- invasive transforaminal lumbar interbody fusion in patients with grade-1 degenerative spondylolisthesis
}

$\mathrm{Da} \mathrm{He}$

Beijing Jishuitan Hospital

Wei He

Beijing Jishuitan Hospital

Yajun Liu

Beijing Jishuitan Hospital

\section{Yuqing Sun}

Beijing Jishuitan Hospital

\section{Yonggang Xing}

Beijing Jishuitan Hospital

\section{Zhao Lang}

Beijing Jishuitan Hospital

Wei Tian (D2602248833@qq.com )

Beijing Jishuitan Hospital

\section{Research article}

Keywords: oblique lumbar interbody fusion, percutaneous pedicle screw fixation, spondylolisthesis, minimally invasive, transforaminal lumbar interbody fusion

Posted Date: January 21st, 2020

DOI: https://doi.org/10.21203/rs.2.21425/v1

License: (c) (i) This work is licensed under a Creative Commons Attribution 4.0 International License.

Read Full License 


\section{Abstract}

Background: The most effective lumbar interbody fusion (LIF) technique for degenerative spondylolisthesis remains controversial.

Methods: This is a retrospective study. This comparative analysis included consecutive patients with grade-1 degenerative spondylolisthesis who underwent oblique LIF (OLIF) or minimally-invasive transforaminal LIF (MI-TLIF) at the Department of Spine Surgery, our hospital(January 2016 to August 2017). Patient satisfaction (Japanese Orthopaedic Association score), visual analog scale (VAS) scores for back and leg pain, Oswestry disability index (ODI), radiographic outcomes including anterior/posterior disc heights (ADH/PDH), foraminal height $(\mathrm{FH})$, foraminal width (FW), cage subsidence, cage retropulsion and fusion rate were assessed during a 2-year follow-up.

Results: The OLIF and MI-TLIF groups comprised 36 patients (age, $52.1 \pm 7.2$ years; 27 women) and 45 patients (age, $48.4 \pm 14.4$ years; 24 women) respectively. Satisfaction rates at 2 years exceeded $90 \%$ in both groups. The OLIF group had less intraoperative blood loss ( $140 \pm 36$ vs. $233 \pm 62 \mathrm{~mL}$ ), lower back pain VAS score ( $2.42 \pm 0.81$ vs. $3.38 \pm 0.47)$ and ODI score $(20.47 \pm 2.53$ vs. $27.31 \pm 3.71)$ at 3 months (with trends toward lower values at 2 years), but higher leg pain VAS scores at all postoperative time points than the MI-TLIF group (all P<0.001). ADH, PDH, FD and FW improved in both groups after surgery. At 2 years, the OLIF group had a higher rate of Bridwell grade-I fusion ( $100 \%$ vs. $88.9 \%, P=0.046)$ and lower incidences of cage subsidence $(8.33 \%$ vs. $46.67 \%, P<0.001)$ and retropulsion ( $0 \%$ vs. $6.67 \%, P=0.046)$.

Conclusions: In patients with grade-I spondylolisthesis, OLIF was associated with less blood loss and better improvements in some clinical(VAS for back pain and ODI) and radiologic outcomes than MI-TLIF, although patients in the OLIF group had higher leg pain VAS scores than patients in the MI-TLIF group.

\section{Background}

Degenerative spondylolisthesis's symptoms include back pain, leg pain and neurogenic claudication[1]. Conventional open procedures such as anterior lumbar interbody fusion (ALIF), posterior lumbar interbody fusion (PLIF) and transforaminal lumbar interbody fusion (TLIF) have been applied with successful outcomes, although each technique has its advantages and disadvantages[2].

Emerging minimally-invasive techniques, such as minimally-invasive TLIF (MI-TLIF) and oblique lumbar interbody fusion (OLIF), are progressively changing the treatment modalities for patients suffering from degenerative lumbar diseases. MI-TLIF achieves direct decompression of spinal neural elements and was first described (using a tubular retractor) by Foley and colleagues in 2003[3]. OLIF was first introduced in 2012[4], and its primary surgical goal is to implant the largest possible interbody cage within the confines of the surgical exposure to facilitate fusion rates, preserve posterior column structure, reduce paraspinal muscle trauma, maximize segmental lordosis and correct sagittal imbalance[5]. 
No previous studies have directly compared the clinical and radiographic outcomes of OLIF and MI-TLIF in patients with spondylolisthesis. We carried out a retrospective analysis of our experiences of OLIF and MI-TLIF in order to compare the clinical and radiologic outcomes of these two techniques.

\section{Methods}

\subsection{Study design and participants}

This was a retrospective analysis of patients with spondylolisthesis who underwent one of the two surgical procedures at our Hospital between January 2016 and August 2017. The inclusion criteria were: 1) spondylolisthesis of Meyerding grade 1 (slippage $\leq 25 \%$ ) at the L4-L5 level was confirmed by radiography $(1) ; 2$ ) surgery was indicated due to symptoms of mechanical low back pain that had not been relieved after more than 6 months of conservative treatment; 3 ) follow-up data were available for a minimum of 24 months. The exclusion criteria were: 1) spinal canal stenosis; 2) caudaequina syndrome; 3) spinal tumor; 4) spinal infection; 5) spinal fracture; and 6) previous surgery at the L4-L5 level.

For the analysis, the patients were divided into an OLIF group and a MI-TLIF group based on the surgical technique used. The surgical method had been selected by the patient after they had been provided with information about the potential advantages and disadvantages of each approach.

The study was approved by the ethics committee of our Hospital (This research has been approved by the IRB of the authors' affiliated institutions), and informed consent was waived due to the retrospective design of the study.

\subsection{Surgical techniques}

OLIF and MI-TLIF were performed under general anesthesia. The same chief surgeon, who had 24 years of experience in spinal surgery, performed all the operations. All patients were discharged 7 days after surgery.

OLIF was performed using a standard procedure[6]. Briefly, a 4-cm skin incision was made $6-10 \mathrm{~cm}$ anterior to the mid-portion of the marked disc. The retroperitoneal space was approached via blunt dissection, and the peritoneum was mobilized anteriorly to expose the anatomical oblique lateral corridor. An intervertebral cage (12 mm high, $50 \mathrm{~mm}$ long and $18 \mathrm{~mm}$ wide; $6^{\circ}$ lordotic; $3.27 \mathrm{cc}$ graft volume; Clydesdale Spinal System, Medtronic, Memphis, TN, USA) filled with demineralized bone matrix (DBM) (Wright Medical Technology Inc., Arlington, TN, USA) was inserted. Subsequently, the patient was placed in the prone position to undergo posterior bilateral percutaneous pedicle screw fixation (CD Horizon Solera Voyager Spinal System, Medtronic). None of the patients in the OLIF group underwent additional laminectomy at the level of the spondylolisthesis. 
MI-TLIF was carried out using a standard method[7]. Unilateral MI-TLIF was performed with the aid of a microscope, using a straight cage (12 mm high, $26 \mathrm{~mm}$ long and $10 \mathrm{~mm}$ wide; $0^{\circ}$ lordotic; $0.90 \mathrm{cc}$ graft volume; Capstone Peek Spinal System, Medtronic) filled with demineralized bone matrix (DBM) (Wright Medical Technology Inc., Arlington, TN, USA) was inserted. Posterior bilateral percutaneous pedicle screw fixation (CD Horizon Solera Voyager Spinal System, Medtronic) was carried out in all patients.

\subsection{Demographic and operative data}

The following demographic and operative data were extracted from the medical records: sex, ageand intraoperative blood loss.

\subsection{Follow-up and outcome measures}

The following assessments were made before surgery and postoperatively at 1 week, 3 months and 2 years: visual analog scale (VAS) scores for back pain and leg pain; Oswestry disability index (ODI, version 2.0)[8]; anteroposterior (AP)/lateral radiography and flexion-extension radiography; computed tomography (CT); and magnetic resonance imaging (MRI). Patient satisfaction with treatment was determined using the Japanese Orthopaedic Association (JOA) scoring system[9]. Patient satisfaction with the clinical effect (with focus on the symptoms in the back and lower limbs) was graded as satisfactory, acceptable or very unsatisfactory. The ODI scoring system was used to evaluate the treatment effect on overall quality of life. The VAS scores of the lower limbs were analyzed to evaluate the effects of treatment on lower limb symptoms. In addition, the occurrence of any complications was recorded.

Evaluation of cage position was based on the imaging investigations. Cage subsidence was defined to be present if a cage was observed to sink into an adjacent vertebral body by $>2 \mathrm{~mm}$, based on comparisons with previous CT images[10]. Cage migration was defined as posterior movement of the cage by $\geq 3 \mathrm{~mm}$ compared with the immediate postoperative state.

Radiologic measurements included: (1) Disc height(DH) including anterior disc height(ADH) and posterior disc height(PDH), (2) foraminal height (FH), (3) foraminal width (FW)and (4) fusion rate. ADH/PDH was defined as the distance of the anterior/posterior position from the upper to the lower endplate of the L4-5 level. FH was defined as the distance from the lower position of the pedicle of $L 4$ to the upper position of the pedicle of L5. FW was defined as the distance from the lower posterior horn of the vertebral body of L4 to the vertex of the superior joint of L5. Fusion grading criteria were based on the Bridwellinterbody fusion grading system[11]. Fusion rate was evaluated postoperatively at 3 months and 2 years. 
2.5 Statistical analysis Continuous data are presented as mean \pm standard deviation and were compared between groups using the independent sample t-test. Categorical data are presented as $n(\%)$ and were compared between groups using the Pearson chi-squared test or Fisher's exact test. Repetitive measurement and analysis of variance was employed in the analysis of ODI, back pain VAS score and leg pain VAS score. Statistical analyses were performed using SPSS 18.0 (SPSS Inc., Chicago, IL, USA). Statistical significance was defined as $P<0.05$.

\section{Results}

\subsection{Baseline clinical characteristics}

A total of 81 patients were included in the analysis, and their baseline clinical characteristics are presented in Table 1. There was significantly less intraoperative blood loss in the OLIF group than in the MI-TLIF group (140 $\pm 36 \mathrm{ml}$ vs. $233 \pm 62 \mathrm{ml}, P<0.001)$.

\subsection{Follow-up}

At 24 months, 76/81 of patients (93.8\%) returned to our hospital for their follow-up visit. Follow-up was conducted by telephone for the 5 patients who lived too far from our hospital to return at 24 months, and their 24-month CT and MRI scans (obtained at a more local institution) were sent to our hospital for analysis. Thus, follow-up data were available for all 81 patients.

\subsection{Clinical outcomes}

Patient satisfaction rate 2 years after surgery (Table 2) was more than $90 \%$ in both groups and not significantly different between the OLIF group (91.7\%) and MI-TLIF group (91.1\%). Preoperatively, there were no significant differences between the OLIF and MI-TLIF groups in back pain VAS score or ODI score (Table 2). Both groups showed progressive improvements in all clinical outcome scores during postoperative follow-up, as compared with preoperative values. Back pain VAS score was significantly lower in the OLIF group than in the MI-TLIF group at 3 months after surgery $(2.42 \pm 0.81$ vs. $3.38 \pm 0.47$, $P<0.001)$, and there was also a trend toward a lower value in the OLIF group at 2 years $(0.86 \pm 0.64 \mathrm{vs}$. $1.07 \pm 0.25, P=0.052$; Table 2). ODI score was also lower in the OLIF group than in the MI-TLIF group at 1 week (33.61 \pm 2.10 vs. $34.75 \pm 2.16, P=0.019)$ and 3 months $(20.47 \pm 2.53$ vs. $27.31 \pm 3.71, P<0.001)$, with a trend toward a lower value at 2 years (14.89 \pm 1.93 vs. $15.82 \pm 1.45, P=0.074$; Table 2$)$.

\subsection{Radiologic outcomes}


ADH was significantly higher in the OLIF group than in the MI-TLIF group at all postoperative time points ( $P=0.015$ at 1 week, $P=0.014$ at 3 months and $P=0.024$ at 2 years) despite being significantly lower preoperatively $(P<0.001$; Table 3$)$.

PDH was significantly higher in the MI-TLIF group than in the OLIF group preoperatively $(P=0.001)$ and at 1 week $(P<0.001)$ and 3 months $(P=0.003)$ postoperatively (Table 3$)$. However, PDH did not differ between groups at 2 years postoperatively (Table 3 ).

FH and FW showed improvements after surgery in both groups (Table 3). FH did not differ significantly between the OLIF and MI-TLIF groups postoperatively (Table 3). The cage used for the OLIF group (50 $\mathrm{mm}$ long, $18 \mathrm{~mm}$ wide and $12 \mathrm{~mm}$ in height) had a larger cross-sectional area than that used for the MITLIF group(26 mm long, $10 \mathrm{~mm}$ wide and $12 \mathrm{~mm}$ in height). However, FW was significantly higher in the MI-TLIF group than in the OLIF group preoperatively $(P=0.002)$ and at 1 week $(P=0.003), 3$ months $(P=$ $0.002)$ and 2 years $(P=0.004)$ postoperatively (Table 3$)$.

The rate of complete fusion (grade I according to Bridwell's criteria) was significantly higher in the OLIF group than in the MI-TLIF group at 3 months $(83.3 \%$ vs. $62.2 \%, P=0.042)$ and at 24 months $(100 \%$ vs. $88.9 \%, P=0.046)$.

\subsection{Cage position}

Cage subsidence occurred more commonly in the MI-TLIF group than in the OLIF group at 3 months postoperatively $(35.6 \%$ vs. $8.3 \%, P=0.003)$ and at 24 months postoperatively $(46.7 \%$ vs. $8.3 \%, P<0.001$; Table 4). In addition, cage retropulsion did not occur in the OLIF group during the 2-year follow-up but was observed in 3 patients $(6.7 \%)$ in the MI-TLIF group $(P=0.046$; Table 4$)$.

\subsection{Complications}

The intraoperative and postoperative complications are listed in Table 4. In the OLIF group, 3 cases of L4 segmental artery injury were noticed during surgery, and rapid hemostasis was achieved with a hemoclip. Five patients (13.9\%) in the OLIF group had sympathetic injury and four patients $(11.1 \%)$ had leg weakness/numbness which were transient in nature and recovered within the first 3 months after surgery without the need for intervention (Table 4).

In the MI-TLIF group, 2 patients (4.4\%) suffered intraoperative dural tears during nerve decompression and developed cerebrospinal fluid leak. Three patients in the MI-TLIF group complained of leg pain/numbness at 24 months after surgery. These 3 patients had cage retropulsion and a fusion grade of $I V$, and their pain resolved after revision surgery to reinsert the cage and compress the adjacent vertebrae. 
Two patients in the MI-TLIF group suffered right L5 root palsy due to a local hematoma but recovered within 3 months.

\section{Discussion}

MI-TLIF is a technically challenging operation carried out in a limited working space. A study reported that the total complication rate was $8.11 \%[12]$, which was lower than previously described rates of $30.77 \%[13]$ and $10.47 \%[14]$.A meta-analysis of 5454 patients published in 2015 concluded that the complication rate for MI-TLIF was $19.2 \%$ overall, $2.2 \%$ for neurologic deficits and $3.6 \%$ for intraoperative complications[15].

OLIF utilizes the ante-psoas muscle approach to exert a different mechanism of action, providing indirect neural decompression by expansion of the bony neuroforamen and distraction of the ligamentous stenosis of the central canal. Mehren et al. reported that the overall incidence of intraoperative and perioperative complications in 812 patients who underwent OLIF was $3.7 \%$, with a neurologic complication rate of $0.37 \%[16]$. In experiments in cadavers, Davis et al. investigated the oblique lateral corridor between the peritoneum and psoas muscle without dissecting or traversing the psoas muscle and concluded that the corridor provides safe and easy access to the L2-S1 intervertebral discs with minimal psoas retraction[17]. Most clinicians consider OLIF to be relatively safe. In a meta-analysis of 1453 patients published in 2017, the overall complication rate associated with OLIF was $11.4 \%$, and the incidences of neurologic deficits and intraoperative complications was $4.2 \%$ and $1.5 \%$, respectively[6].

Indeed, the back pain VAS score at 3 months was significantly lower in the OLIF group than in the MI-TLIF group. Milder back pain symptoms in the OLIF group at 3 months may have been due to less iatrogenic violation of the posterior lumbar elements than that which occurs after MI-TLIF (Fig. 1). An additional advantage of OLIF over MI-TLIF is that the cage can achieve greater sagittal angle improvement by being implanted in the front third of the disc, which helps relieve tension in the paravertebral muscles. It was also notable that the ODI score at 3 months was significantly lower in the OLIF group than in the MI-TLIF group, suggesting that OLIF may have advantages over MI-TLIF with regard to achieving the intended surgical outcomes.

The incidence of cage subsidence as a complication of lateral lumbar interbody fusion (LLIF) is $10.84 \%$ [15]. In this study, 3 cases of endplate injury occurred in the OLIF group, and CT examination at the 2-year follow-up confirmed that 3 cages (8.3\%) had shown subsidence. By contrast, the 2-year follow-up revealed cage subsidence in 21 patients $(46.7 \%)$ in the MI-TLIF group. Accompanying phenomena observed in the MI-TLIF group were postoperative decreases in PDH and FH between 1 week and 2 years. One possible explanation for the above results is that the intervertebral space occupied by the cage was wider in the OLIF group (cross-sectional cage area of $900 \mathrm{~mm}^{2}$ ) than in the MI-TLIF group (crosssectional cage area of $260 \mathrm{~mm}^{2}$ ). The wider footprint of the cage used for OLIF may provide a more effective biological environment for the fusion process, reducing the chances of cage subsidence. 
Consistent with this proposal, Tohmeh et al. found that severe subsidence ( $\geq 4 \mathrm{~mm}$ ) was more likely for a $50-\mathrm{mm}$ cage than for a $60-\mathrm{mm}$ cage[18]. A second possible reason is that the OLIF cage is implanted more securely on the dense ring apophysis because it runs through both sides of the endplate and is located anteriorly, which is the strongest part of the endplate, whereas the MI-TLIF cage is mostly located in the central, weaker part of the endplate (Fig. 2). Xu et al. reported a significantly higher rate of endplate damage with the transforaminal approach (48\%) than with the lateral approach (4\%)[19]. Among 178 patients followed-up for 25 months after LLIF, Malham et al. identified 13 patients ( 14 operative segments) with cage subsidence, with all cases occurring in the inferior endplates[20]. It is important that surgeons are careful during preparation of the endplates to avoid endplate injury.

Cage retropulsion may result in the loss of lumbar lordosis, a narrowing of the disc space and foraminae, direct compression of the nerve roots and a lower fusion rate[21, 22]. In this study, we found a higher incidence of cage retropulsion in the MI-TLIF group than in the OLIF group (no cases of cage retropulsion). We consider that the use of a lateral annular incision maintained the integrity of the posterior longitudinal ligament and posterior annulus in the OLIF group, which can theoretically prevent cage retropulsion. Buttermann et al. proposed that proper annular tension may reduce the risk of implant migration[23]. In addition, the sacral slope and pelvic incidence were greater in these 3 patients than in the other patients. Several studies have reported that interbody implants at L5-S1 are at greater risk of retropulsion[21, 24].

The most common complications of OLIF are lumbar sympathetic trunk injury and segmental artery injury. Lumbar sympathetic trunk injury is usually characterized by elevated skin temperature, reduced perspiration, paresthesia, skin discoloration, swelling of the lower limb on the surgical side. Neurogenic pain is aggravated at night[25]. In this study, the probability of lumbar sympathetic trunk injury was higher for OLIF than for MI-TLIF. In the OLIF group, there were 5 cases $(13.9 \%)$ of anterolateral thigh pain or numbness, all of which were due to sympathetic chain injury, and 3 cases $(8.3 \%)$ of segmental artery injury. Other authors have described similar findings. Hrabaleka et al. showed that the symptoms of sympathetic nerve injury can last up to 53 months[25]. Jin et al. reported that complications of OLIF occurred in 3 of 21 patients (leg paresthesia in 2 and local hematoma in 1)[26]. One possible reason for the occurrence of lumbar sympathetic trunk injury during OLIF is that if the incision is not properly planned, excessive pressure on the psoas could result in injury to the genitofemoral nerve and sympathetic chain. Due to the specific anatomic path used for OLIF, the possibility of nerve root injury during surgery is lower. We suggest that measures to minimize nerve injury and reduce neurologic symptoms should include a clear visual field, careful performance of the operation, especially during separation of the psoas major and vascular sheath, and avoidance of electric coagulation/use of an electric knife.

This study has several limitations. First, this was a retrospective study, so the analysis may have been affected by information bias or selection bias. Second, this was a single-center study with a small sample size. Third, the follow-up time was limited to 2 years, so longer-term outcomes were not evaluated. 


\section{Conclusions}

In patients with grade-I spondylolisthesis, OLIF was associated with less blood loss and better improvements in some clinical(VAS for back pain and ODI) and radiologic outcomes than MI-TLIF, although patients in the OLIF group had higher leg pain VAS scores than patients in the MI-TLIF group. Because the biggest limitation of OLIF is indirect decompression, the ability to relieve symptoms of lower limbs is limited. Our advice is to choose patients with low back pain as the main symptoms, accompanied by mild or no leg symptoms before operation. Moreover, during OLIF surgery, particular attention should be paid to the lumbar sympathetic trunk to reduce postoperative neurologic complications.

\section{Abbreviations}

Oblique lateral interbody fusion (OLIF), percutaneous pedicle screws fixation (PPSF), Computed tomography (CT), magnetic resonance imaging (MRI), anterior lumbar interbody fusion (ALIF), posterior lumbar interbody fusion (PLIF), transforaminal lumbar interbody fusion (TLIF), minimally-invasive(MI), visual analog scale (VAS), Oswestry disability index (ODI), anterior/posterior disc heights (ADH/PDH), foraminal height $(\mathrm{FH})$, foraminal width $(\mathrm{FW})$, demineralized bone matrix (DBM).

\section{Declarations}

Ethics approval and consent to participate

The study was approved by the ethics committee of the Beijing Jishuitan hospital. Informed consent was waived because of the retrospective nature of the study.

Consent for publication

Not applicable.

Availability of data and materials

The datasets used and/or analyzed during the current study are available from the corresponding author on reasonable request.

Competing interests

All authors declare that they have no competing interests.

Funding 
This study was funded by Beijing Jishuitan Hospital "Nova Program"

(\#XKXX201808),Beijing Hospital Authority Youth Program (\#QML20190403), Beijing Jishuitan Hospital "Medical and Engineering Intersection Project” (\#YGQ-201924), and Beijing “Ten, Hundred, Thousand” Medical Talents Project: “Application of computer navigation-assisted minimally invasive surgery and traditional surgery in lumbar degenerative disease".

Authors' contributions

D H and W H carried out the studies and drafted the manuscript. YJ L, YQ S and YG X performed the statistical analysis and participated in its design. Z L collected data. W T participated in acquisition, analysis, or interpretation of data and drafted the manuscript. All authors read and approved the final manuscript.

Acknowledgment

None.

\section{References}

1. Kalichman L, Hunter DJ. Diagnosis and conservative management of degenerative lumbar spondylolisthesis. Eur Spine J. 2008 Mar;17(3):327-35.

2. Mobbs RJ, Phan K, Malham G, Seex K, Rao PJ. Lumbar interbody fusion: techniques, indications and comparison of interbody fusion options including PLIF, TLIF, MI-TLIF, OLIF/ATP, LLIF and ALIF. J Spine Surg. 2015 Dec;1(1):2-18.

3. Foley KT, Holly LT, Schwender JD. Minimally invasive lumbar fusion. Spine. 2003 Aug 1;28(15 Suppl):S2635.

4. Silvestre C, Mac-Thiong JM, Hilmi R, Roussouly P. Complications and Morbidities of Mini-open Anterior Retroperitoneal Lumbar Interbody Fusion: Oblique Lumbar Interbody Fusion in 179 Patients. Asian Spine J. 2012 Jun;6(2):89-97.

5. Fujibayashi S, Hynes RA, Otsuki B, Kimura H, Takemoto M, Matsuda S. Effect of indirect neural decompression through oblique lateral interbody fusion for degenerative lumbar disease. Spine. 2015 Feb $1 ; 40(3): E 175-82$.

Page $10 / 18$ 
6. Li JX, Phan K, Mobbs R. Oblique Lumbar Interbody Fusion: Technical Aspects, Operative Outcomes, and Complications. World Neurosurg. 2017 Feb;98:113-23.

7. Wong AP, Smith ZA, Stadler JA, 3rd, Hu XY, Yan JZ, Li XF, Lee JH, Khoo LT. Minimally invasive transforaminal lumbar interbody fusion (MI-TLIF): surgical technique, long-term 4-year prospective outcomes, and complications compared with an open TLIF cohort. Neurosurg Clin N Am. 2014 Apr;25(2):279304.

8. Roland M, Fairbank J. The Roland-Morris Disability Questionnaire and the Oswestry Disability Questionnaire. Spine. 2000 Dec 15;25(24):3115-24.

9. Fujimori T, Okuda S, Iwasaki M, Yamasaki R, Maeno T, Yamashita T, Matsumoto T, Wada E, Oda T. Validity of the Japanese Orthopaedic Association scoring system based on patient-reported improvement after posterior lumbar interbody fusion. J Spine. 2016 Jun;16(6):728-36.

10. Kim MC, Chung HT, Cho JL, Kim DJ, Chung NS. Subsidence of polyetheretherketone cage after minimally invasive transforaminal lumbar interbody fusion. J Spinal Disord Tech. 2013 Apr;26(2):87-92.

11. Bridwell KH, Lenke LG, McEnery KW, Baldus C, Blanke K. Anterior fresh frozen structural allografts in the thoracic and lumbar spine. Do they work if combined with posterior fusion and instrumentation in adult patients with kyphosis or anterior column defects? Spine. 1995 Jun 15;20(12):1410-8.

12. Xu YF, Le XF, Tian W, Liu B, Li Q, Zhang GL, Liu YJ, Yuan Q, He D, Mao JP, Xiao B, Lang Z, Han XG, Jin PH. Computer-assisted, minimally invasive transforaminal lumbar interbody fusion: One surgeon's learning curve A STROBE-compliant article. Medicine. 2018 Jul;97(27):e11423.

13. Nandyala SV, Fineberg SJ, Pelton M, Singh K. Minimally invasive transforaminal lumbar interbody fusion: one surgeon's learning curve. JSpine. 2014 Aug 1;14(8):1460-5.

14. Lee JC, Jang HD, Shin BJ. Learning curve and clinical outcomes of minimally invasive transforaminal lumbar interbody fusion: our experience in 86 consecutive cases. Spine. 2012 Aug 15;37(18):1548-57.

15. Joseph JR, Smith BW, La Marca F, Park P. Comparison of complication rates of minimally invasive transforaminal lumbar interbody fusion and lateral lumbar interbody fusion: a systematic review of the literature. Neurosurg Focus. 2015 Oct;39(4):E4.

16. Mehren C, Mayer HM, Zandanell C, Siepe CJ, Korge A. Reply to the Letter to the Editor: The Oblique Anterolateral Approach to the Lumbar Spine Provides Access to the Lumbar Spine With Few Early Complications. Clin Orthop Relat Res. 2017 Jan;475(1):289-90.

17. Davis TT, Hynes RA, Fung DA, Spann SW, MacMillan M, Kwon B, Liu J, Acosta F, Drochner TE. Retroperitoneal oblique corridor to the L2-S1 intervertebral discs in the lateral position: an anatomic study. J Neurosurg Spine. 2014 Nov;21(5):785-93.

18. Tohmeh AG, Khorsand D, Watson B, Zielinski X. Radiographical and clinical evaluation of extreme lateral interbody fusion: effects of cage size and instrumentation type with a minimum of 1-year follow-up. 
Spine. 2014 Dec 15;39(26):E1582-91.

19. Xu DS, Bach K, Uribe JS. Minimally invasive anterior and lateral transpsoas approaches for closed reduction of grade II spondylolisthesis: initial clinical and radiographic experience. Neurosurg Focus. 2018 Jan;44(1):E4.

20. Malham GM, Parker RM, Blecher CM, Seex KA. Assessment and classification of subsidence after lateral interbody fusion using serial computed tomography. J Neurosurg Spine. 2015 Nov;23(5):589-97.

21. Kimura H, Shikata J, Odate S, Soeda T, Yamamura S. Risk factors for cage retropulsion after posterior lumbar interbody fusion: analysis of 1070 cases. Spine. 2012 Jun 1;37(13):1164-9.

22. Abbushi A, Cabraja M, Thomale UW, Woiciechowsky C, Kroppenstedt SN. The influence of cage positioning and cage type on cage migration and fusion rates in patients with monosegmental posterior lumbar interbody fusion and posterior fixation. Eur Spine J. 2009 Nov;18(11):1621-8.

23. Buttermann GR, Beaubien BP, Freeman AL, Stoll JE, Chappuis JL. Interbody device endplate engagement effects on motion segment biomechanics. J Spine. 2009 Jul;9(7):564-73.

24. Aoki Y, Yamagata M, Nakajima F, Ikeda Y, Shimizu K, Yoshihara M, Iwasaki J, Toyone T, Nakagawa K, Nakajima A, Takahashi K, Ohtori S. Examining risk factors for posterior migration of fusion cages following transforaminal lumbar interbody fusion: a possible limitation of unilateral pedicle screw fixation. J Neurosurg Spine. 2010 Sep;13(3):381-7.

25. Hrabalek L, Sternbersky J, Adamus M. Risk of sympathectomy after anterior and lateral lumbar interbody fusion procedures. Biomed Pap Med Fac Univ Palacky 2015 Jun;159(2):318-26.

26. Jin J, Ryu KS, Hur JW, Seong JH, Kim JS, Cho HJ. Comparative Study of the Difference of Perioperative Complication and Radiologic Results: MIS-DLIF (Minimally Invasive Direct Lateral Lumbar Interbody Fusion) Versus MIS-OLIF (Minimally Invasive Oblique Lateral Lumbar Interbody Fusion). Clin Spine Surg. 2018 Feb;31(1):31-6.

\section{Tables}

Table 1. Baseline and operative characteristics of the patients in the two groups. 


\begin{tabular}{lccc}
\hline Characteristic & OLIF group & MI-TLIF group & $P$ \\
& $(n=36)$ & $(n=45)$ & \\
\hline Sex & & & $0.043^{\#}$ \\
Male & $9(25.0 \%)$ & $21(46.7 \%)$ & \\
Female & $27(75.0 \%)$ & $24(53.3 \%)$ & \\
\hline Age (years) & $52.1 \pm 7.2$ & $48.4 \pm 14.4$ & $0.097^{\S}$ \\
\hline Intraoperative blood loss $(\mathrm{mL})$ & $140 \pm 36$ & $233 \pm 62$ & $<0.001^{\S}$ \\
\hline
\end{tabular}

Data are presented as $n(\%)$ or mean \pm standard deviation. MI-TLIF: minimally-invasive transforaminal lumbar interbody fusion; OLIF: oblique lumbar interbody fusion. ${ }^{\#}$ chi-squared test; ${ }^{\S}$ t-test.

Table 2. Comparison of clinical outcome scores between the two groups.

\begin{tabular}{|c|c|c|c|}
\hline Measure & $\begin{array}{l}\text { OLIF group } \\
\qquad(n=36)\end{array}$ & $\begin{array}{l}\text { MI-TLIF group } \\
\qquad(n=45)\end{array}$ & $P$ \\
\hline \multicolumn{4}{|l|}{ Back pain VAS score } \\
\hline Preoperative & $8.3 \pm 0.7$ & $8.2 \pm 0.3$ & $0.510^{\S}$ \\
\hline Postoperative, 1 week & $5.6 \pm 0.8$ & $5.4 \pm 0.6$ & $0.280^{\S}$ \\
\hline Postoperative, 3 months & $2.4 \pm 0.8$ & $3.3 \pm 0.4$ & $0.000^{\S}$ \\
\hline Postoperative, 2 years & $0.8 \pm 0.6$ & $1.0 \pm 0.2$ & $0.052^{\S}$ \\
\hline \multicolumn{4}{|l|}{ Leg pain VAS score } \\
\hline Preoperative & $5.5 \pm 0.9$ & $7.7 \pm 0.8$ & $<0.001^{\S}$ \\
\hline Postoperative, 1 week & $3.2 \pm 0.7$ & $2.0 \pm 0.6$ & $<0.001^{\S}$ \\
\hline Postoperative, 3 months & $2.8 \pm 0.9$ & $1.9 \pm 0.7$ & $<0.001^{\S}$ \\
\hline Postoperative, 2 years & $2.2 \pm 0.5$ & $1.4 \pm 0.4$ & $<0.001^{\S}$ \\
\hline \multicolumn{4}{|l|}{ Oswestry disability index score } \\
\hline Preoperative & $44.6 \pm 3.3$ & $45.2 \pm 1.5$ & $0.312^{\S}$ \\
\hline Postoperative, 1 week & $33.6 \pm 2.1$ & $34.7 \pm 2.1$ & $0.019^{\S}$ \\
\hline Postoperative, 3 months & $20.4 \pm 2.5$ & $27.3 \pm 3.7$ & $<0.001^{\S}$ \\
\hline Postoperative, 2 years & $14.8 \pm 1.9$ & $15.8 \pm 1.4$ & $0.074^{\S}$ \\
\hline Patient satisfaction at 2 years & & & $1.000^{\#}$ \\
\hline Satisfied & $33(91.7 \%)$ & $41(91.1 \%)$ & \\
\hline Not satisfied & $3(8.3 \%)$ & $4(8.9 \%)$ & \\
\hline
\end{tabular}

Data are presented as $n(\%)$ or mean \pm standard deviation. MI-TLIF: minimally-invasive transforaminal lumbar interbody fusion; OLIF: oblique lumbar interbody fusion; VAS: visual analogue scale. Patient satisfaction was determined using the Japanese Orthopaedic Association scoring 
system. ${ }^{\#}$ chi-squared test; ${ }^{\S}$ t-test.

Table 3. Comparison of radiologic outcomes between the two groups. 


\begin{tabular}{|c|c|c|c|}
\hline Measure & $\begin{array}{l}\text { OLIF group } \\
\qquad(n=36)\end{array}$ & $\begin{array}{l}\text { MI-TLIF group } \\
\qquad(n=45)\end{array}$ & $P$ \\
\hline \multicolumn{4}{|l|}{ Anterior disc height (mm) } \\
\hline Preoperative & $4.79 \pm 1.6$ & $7.26 \pm 2.0$ & $<0.001^{\S}$ \\
\hline Postoperative, 1 week & $12.82 \pm 1.1$ & $12.15 \pm 0.9$ & $0.015^{\S}$ \\
\hline Postoperative, 3 months & $12.78 \pm 1.6$ & $12.08 \pm 0.8$ & $0.014^{\S}$ \\
\hline Postoperative, 2 years & $12.44 \pm 1.1$ & $12.00 \pm 0.9$ & $0.024^{\S}$ \\
\hline \multicolumn{4}{|l|}{ Posterior disc height(mm) } \\
\hline Preoperative & $4.59 \pm 2.0$ & $6.07 \pm 1.8$ & $0.001^{\S}$ \\
\hline Postoperative, 1 week & $8.53 \pm 1.7$ & $11.32 \pm 0.9$ & $<0.001^{\S}$ \\
\hline Postoperative, 3 months & $8.32 \pm 1.6$ & $9.23 \pm 0.6$ & $0.003^{\S}$ \\
\hline Postoperative, 2 years & $8.07 \pm 1.6$ & $8.12 \pm 0.6$ & $0.868^{\S}$ \\
\hline \multicolumn{4}{|l|}{ Foraminal height (mm) } \\
\hline Preoperative & $12.37 \pm 3.6$ & $13.13 \pm 2.9$ & $0.278^{\S}$ \\
\hline Postoperative, 1 week & $16.27 \pm 4.0$ & $17.01 \pm 2.3$ & $0.322^{\S}$ \\
\hline Postoperative, 3 months & $16.21 \pm 3.8$ & $15.96 \pm 3.0$ & $0.702^{\S}$ \\
\hline Postoperative, 2 years & $16.11 \pm 3.8$ & $14.55 \pm 2.3$ & $0.132^{\S}$ \\
\hline \multicolumn{4}{|l|}{ Foraminal width (mm) } \\
\hline Preoperative & $7.28 \pm 2.2$ & $8.75 \pm 1.2$ & $0.002^{\S}$ \\
\hline Postoperative, 1 week & $9.47 \pm 1.9$ & $10.81 \pm 1.2$ & $0.003^{\S}$ \\
\hline Postoperative, 3 months & $9.35 \pm 1.8$ & $10.71 \pm 2.2$ & $0.002^{\S}$ \\
\hline Postoperative, 2 years & $9.21 \pm 1.9$ & $10.59 \pm 1.2$ & $0.004^{\S}$ \\
\hline \multicolumn{4}{|l|}{ Bridwellinterbody fusion } \\
\hline Postoperative, 3 months & & & $0.042^{\#}$ \\
\hline Grade I & $30(83.3 \%)$ & $28(62.2 \%)$ & \\
\hline Grade II & $4(11.1 \%)$ & $10(22.2 \%)$ & \\
\hline Grade III & $2(5.6 \%)$ & $7(15.6 \%)$ & \\
\hline Grade IV & 0 & 0 & \\
\hline Postoperative, 2 years & & & $0.046^{\#}$ \\
\hline Grade I & $36(100 \%)$ & $40(88.9 \%)$ & \\
\hline Grade II & 0 & $2(4.4 \%)$ & \\
\hline Grade III & 0 & 0 & \\
\hline Grade IV & 0 & $3(6.7 \%)$ & \\
\hline
\end{tabular}


Data are presented as $n(\%)$ or mean \pm standard deviation. MI-TLIF: minimally-invasive transforaminal lumbar interbody fusion; OLIF: oblique lumbar interbody fusion. ${ }^{\#}$ chi-squared test; ${ }^{\S}$ t-test.

Table 4. Complications

\begin{tabular}{|c|c|c|c|}
\hline Complication & $\begin{array}{l}\text { OLIF group } \\
\qquad(n=36)\end{array}$ & $\begin{array}{l}\text { MI-TLIF group } \\
\qquad(n=45)\end{array}$ & $P$ \\
\hline \multicolumn{4}{|l|}{ Intraoperative complications } \\
\hline Segmental artery injury & $3(8.3 \%)$ & 0 & 0.033 \\
\hline Dural tear & 0 & $2(4.4 \%)$ & 0.105 \\
\hline Screw malposition & 0 & 0 & - \\
\hline \multicolumn{4}{|c|}{ Early postoperative complications } \\
\hline Leg weakness/numbness & $4(11.1 \%)$ & $2(4.4 \%)$ & 0.255 \\
\hline Sympathetic chain injury & $5(13.9 \%)$ & 0 & 0.004 \\
\hline Cerebrospinal fluid leak & 0 & $2(4.4 \%)$ & 0.105 \\
\hline Infection & 0 & 0 & \\
\hline Cage subsidence & $3(8.3 \%)$ & $16(35.6 \%)$ & 0.003 \\
\hline Cage retropulsion & 0 & $3(6.7 \%)$ & 0.046 \\
\hline \multicolumn{4}{|c|}{ Late postoperative complications } \\
\hline Leg pain/numbness & 0 & $3(6.7 \%)$ & 0.046 \\
\hline Adjacent segment disease & 0 & 0 & \\
\hline Deep vein thrombosis & 0 & 0 & \\
\hline Cage subsidence & $3(8.3 \%)$ & $21(46.7 \%)$ & $<0.001$ \\
\hline Cage retropulsion & 0 & $3(6.7 \%)$ & 0.046 \\
\hline
\end{tabular}

Data are presented as $n$ (\%). MI-TLIF: minimally-invasive transforaminal lumbar interbody fusion; OLIF: oblique lumbar interbody fusion. $P$-values were determined using the chi-squared test.

\section{Figures}




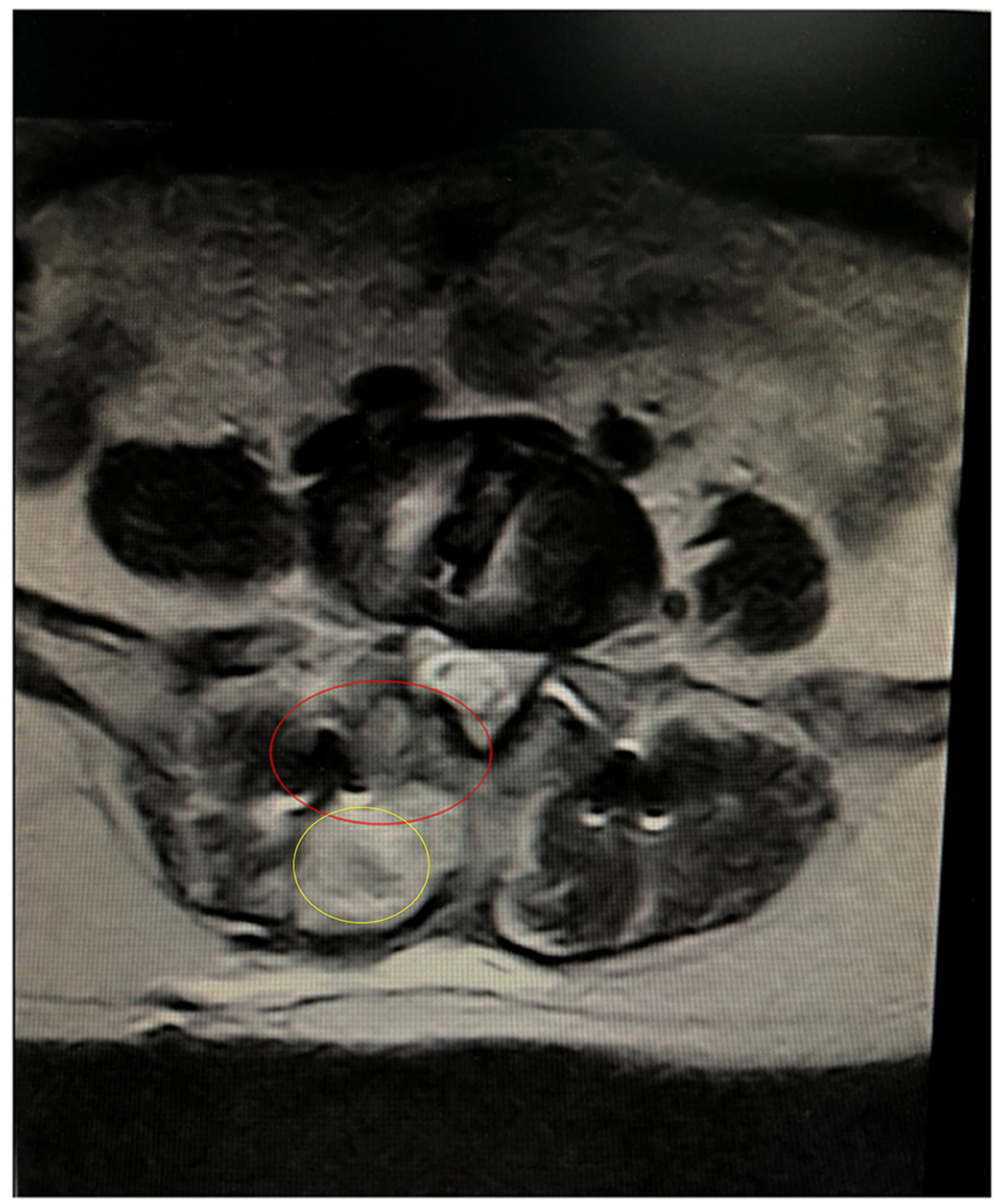

\section{Figure 1}

Tissue injury resulting from minimally-invasive transforaminal lumbar interbody fusion (MI-TLIF). The red circle highlights muscular tissue injury near the spinous process ( 2 years after surgery) caused by the surgical approach during MI-TLIF. 

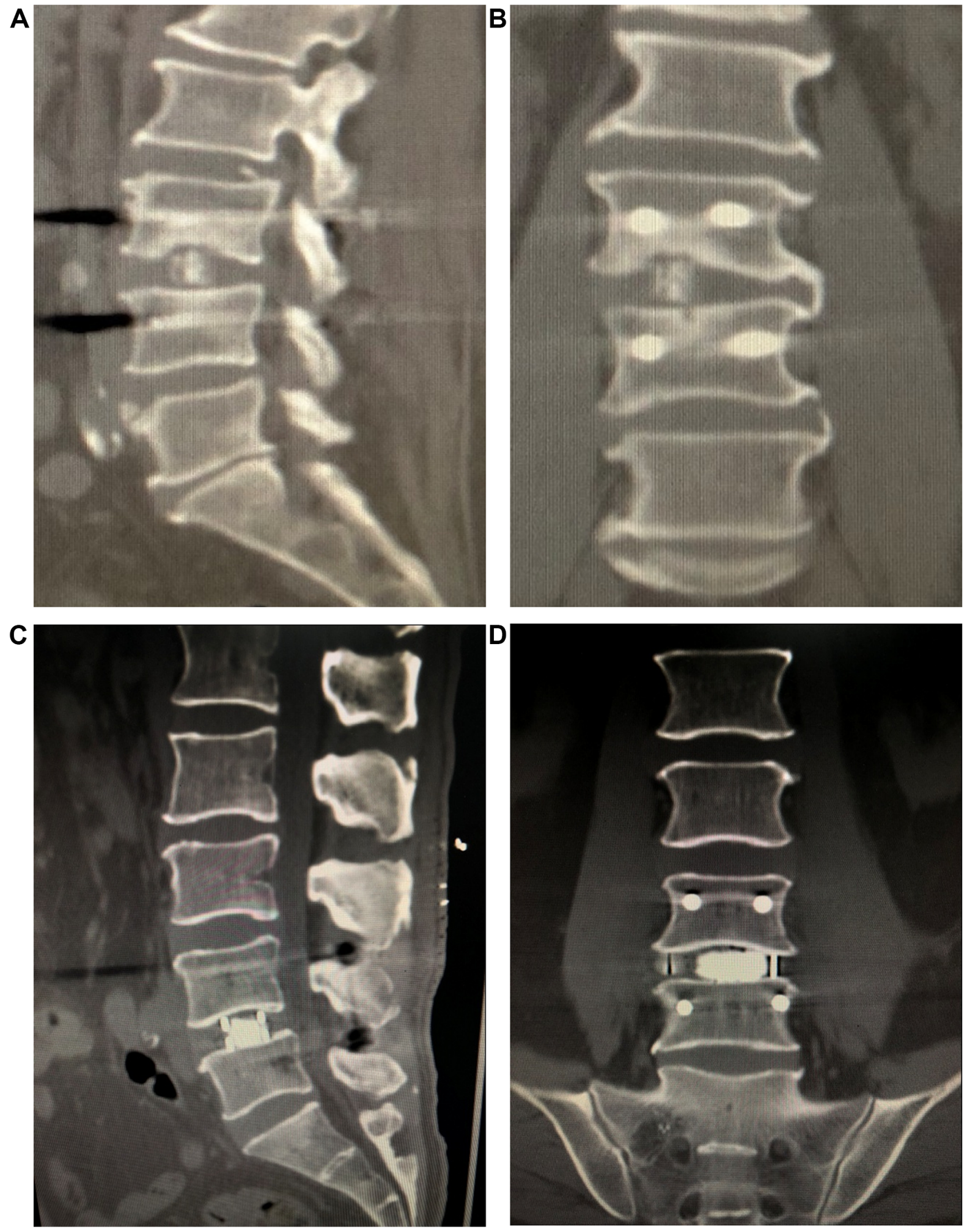

\section{Figure 2}

Computed tomography (CT) images after oblique lumbar interbody fusion (OLIF) and minimally-invasive transforaminal lumbar interbody fusion (MI-TLIF). A. Sagittal CT image after MI-TLIF. B. Anteroposterior image after MI-TLIF. C. Sagittal CT image after OLIF. D. Anteroposterior image after OLIF. 